\title{
Entre ambitions universalistes et concurrences internationales. Retour sur le pari manqué de l'Association pédagogique universelle (1863-1900)
}

Between universalist ambitions and international competition. The failed challenge of the Universal Educational Association (1863-1900)

\section{Alexandre Fontaine}

\section{OpenEdition}

Journals

\section{Édition électronique}

URL : https://journals.openedition.org/histoire-education/2709

DOI : 10.4000/histoire-education.2709

ISSN : 2102-5452

\section{Éditeur}

ENS Éditions

\section{Édition imprimée}

Date de publication : 20 décembre 2013

Pagination : $31-50$

ISBN : $978-2-84788-502-6$

ISSN : 0221-6280

\section{Référence électronique}

Alexandre Fontaine, «Entre ambitions universalistes et concurrences internationales. Retour sur le pari manqué de l'Association pédagogique universelle (1863-1900) », Histoire de l'éducation [En ligne], 139 | 2013, mis en ligne le 15 décembre 2015, consulté le 20 mai 2021. URL : http:// journals.openedition.org/histoire-education/2709; DOI : https://doi.org/10.4000/histoire-education. 2709 


\title{
Entre ambitions universalistes et concurrences internationales. Retour sur le pari manqué de l'Association pédagogique universelle (1863-1900)
}

\author{
Alexandre Fontaine
}

\begin{abstract}
"Si la mission politique et sociale de la Suisse, au milieu des grandes nations qui l'entourent, est une mission de paix, de neutralité, de médiation, de bienfaisance, le rôle de la pédagogie suisse ne serait-il pas de servir d'intermédiaire et de trait d'union entre les systèmes d'éducation du Nord et du Midi? N'est-ce pas là un des caractères essentiels de ce programme de fédération universelle, proclamé à Paris en 1867, discuté et voté à Genève, et dont la réalisation définitive doit faire l'objet de la dernière séance de ce congrès " ${ }^{1}$.
\end{abstract}

S’interrogeant sur les liens étroits mais aussi, au premier abord, paradoxaux entre le monde globalisé que nous connaissons et la réactivation intense des références au national, Anne-Marie Thiesse souligne que l'internationalisme du second $\mathrm{XIX}^{\mathrm{e}}$ siècle "valide le nouveau principe du monde des nations [par lequel] l'internationalité intellectuelle et culturelle est célébrée, mais où dans le même temps sont affirmés des esprits nationaux spécifiques qui divisent l'art et la science en unités radicalement distinctes et concurrentes "². Portée par le pédagogue suisse Alexandre Daguet et la Société des instituteurs de la Suisse romande, l'Association pédagogique universelle, dont la création est décidée en 1872 à Genève, a de fait facilité la mise en réseau, sous diverses formes,

1 Discours d'Alexandre Daguet lors du congrès de Saint-Imier, in Compte-rendu du Ve congrès scolaire de la Société des instituteurs de la Suisse romande tenu à Saint-Imier les 20, 21 et 22 juillet 1874, Saint-Imier, Impr. Ernest Grossniklaus, 1874, p. 64.

2 Anne-Marie Thiesse, "Nations, internationalismes et mondialisation", Romantisme, n 163, 2014, p. 23. 
des actrices et des acteurs de l'Europe pédagogique pendant plus d'un quart de siècle tout en incitant ces derniers à s'engager dans la constitution et la consolidation de structures pédagogiques nationales, régionales ou cantonales. Bien qu'il s'agisse d'une tentative manquée dans le sens où l'Association n'aboutira pas à la constitution d'une structure internationale stable sur le long terme, cette entreprise internationale demeure un maillon constitutif important de l'histoire du mouvement associatif pédagogique européen.

Cet article, qui s'appuie sur une recherche plus large ${ }^{3}$, cherchera dans un premier temps à éclairer la genèse, les enjeux et les mécanismes de mise en place de ce mouvement international encore peu connu. La focale sera ensuite placée sur les diverses relances qui furent tentées suite à l'échec de l'association, que ce soit au travers de la mise en place d'un réseau singulier de la presse d'éducation ou par une réactivation postérieure que l'on doit à Ferdinand Buisson.

\section{I - «Instituteurs de tous les pays, unissez-vous!»}

Dans L'Union ouvrière publié en 1843, soit cinq ans avant le Manifeste du Parti communiste de Marx et Engels, Flora Tristan (1803-1844) exhorte les prolétaires à s'unir afin d'aboutir à l'uunion universelle des ouvriers et des ouvrières " Dès la moitié du XIX ${ }^{\text {e }}$ siècle, les enseignants s'inscrivent également dans une dynamique d'internationalisation, à l'instar des ouvriers qui se fédèrent au sein de l'Association internationale des travailleurs à Londres en 1864, ou encore des scientifiques européens qui se dotent de "nouveaux lieux de savoirs" ${ }^{5}$. La Suisse occupe une place importante dans ce processus, notamment avec la création de la Croix-Rouge en 1864, de l'Union postale universelle, fondée à Berne en 1874, et de la Ligue internationale de la paix et de la liberté portée par les républicains français installés dans le repli helvétique. Jules Barni (1818-1878) qui, avec l'appui d'Edgar Quinet, occupe la chaire de philosophie de l'université de Genève entre 1861 et 1870, rappelle en effet, lors du premier

3 Alexandre Fontaine, Transferts culturels et déclinaisons de la pédagogie européenne. Le cas franco-romand au travers de l'itinéraire d'Alexandre Daguet (1816-1894), thèse de doctorat, universités de Paris 8 et de Fribourg (Suisse), 2013. Cette recherche a bénéficié du soutien du Fonds national suisse de la recherche scientifique, subsides FNS PBFRP1-127988 et 129474.

4 Flora Tristan, L'Union ouvrière, Paris, Chez tous les libraires, 1844 (1 ${ }^{\mathrm{re}}$ éd. 1843), p. 4.

5 Voir Pascale Rabault-Feuerhahn, Wolf Feuerhahn, "La fabrique internationale de la science. Les congrès scientifiques de 1865 à 1945", numéro thématique de la Revue germanique internationale, $\mathrm{n}^{\circ} 12,2010$. 
congrès de la Ligue en septembre 1867 dont il est le vice-président, que le but recherché consiste à jeter les bases d'une vaste confédération devant relier les peuples émancipés du continent, afin d'aboutir à la constitution des "ÉtatsUnis d'Europe». Plus encore, la Confédération helvétique est à considérer, selon Barni, comme un laboratoire miniaturisé d'une Europe que l'on doit construire à son image :

"Notre idée s'appuie, d'ailleurs, sur une réalité vivante qui peut servir à la fois de modèle et de noyau à l'organisation de l'Europe. Je veux parler de la Suisse, où notre ligue a pour cette raison même établi son siège. La Suisse ne comprend-elle pas vingt-deux États indépendants, appartenant à des nationalités et parlant des langues diverses? Et ces vingt-deux États ne forment-ils pas une Confédération qui, en laissant à chacun son autonomie, les unit pour le règlement et la défense de leurs intérêts communs, et entretient entre eux l'harmonie et la paix? Eh bien! C'est ce système qu'il s'agit d'étendre à l'Europe entière. Ce que la Suisse est en petit, il faut que l'Europe le devienne en grand, si elle veut extirper à jamais de son sein le fléau de la guerre et des armées permanentes ${ }^{6}$.

Les enseignants suisses participent également à cette poussée internationaliste et s'associent afin de valoriser leur position et de se construire un "socle commun" en matière d'éducation.

\section{1 - Les instituteurs de la Suisse romande, entre convergences régionales et ambitions universalistes}

Si les régents alémaniques se mobilisent au sein du Schweizerischer Lehrerverein (Société pédagogique de Suisse allemande) dès 1849, les instituteurs romands éprouvent rapidement le besoin de s'unir et de s'autonomiser par rapport à leurs confrères alémaniques. C'est lors de la cinquième réunion du Lehrerverein à Berne, en octobre 1863, que quelques enseignants provenant de la Suisse française, "se sentant un peu perdus au milieu de sept cents collègues germains »", décident de se regrouper afin d'échanger leurs préoccupations pédagogiques. Un comité d'initiative est formé et la fusion des enseignants romands avec ceux du Lehrerverein est un temps envisagée. Le projet est vite délaissé tant la langue allemande demeure un fossé infranchissable :

6 Jules Barni, "Discours", Bulletin officiel du congrès de la paix et de la liberté de 1869, Lausanne, Association typographique, 1869, p.9.

7 Alexandre Daguet, "Rapport sur l'origine et la marche de la Société des instituteurs de la Suisse romande", in Rapport sur la deuxième session de l'assemblée générale des instituteurs de la Suisse romande réunis le 6 août, Fribourg, Impr. Marchand, 1866, p. 7. 
"La Suisse allemande peut déjà nous présenter les bienfaits d'une association pédagogique. Certes, nous n'eussions pas mieux demandé, pour la facilité de notre tâche, que de nous joindre à elle et de profiter ainsi de ce qui existe. Mais, après avoir mûrement étudié la chose, il nous est apparu qu'il serait de toute impossibilité de réunir dans une même assemblée les instituteurs des diverses langues de la Suisse. En effet, il ne peut être question de faire entrer dans une société de langue allemande des hommes qui n'en comprennent pas le premier mot, qui ne pourront ainsi prendre part en connaissance de cause aux délibérations, et dont le nombre d'ailleurs sera toujours dominé par la majorité qui les environnera. Nous pourrons d'ailleurs nous allier en tout temps avec nos frères allemands; ce que nous réclamons ici, c'est le maintien de notre individualité. D’autre part, ce qui importe surtout d'avoir avec eux, c'est une communauté d'intentions. Or, notre programme est le même" ${ }^{8}$.

De ce fait, quelque 250 enseignants réunis en assemblée à Neuchâtel le 26 septembre 1864 fondent la Société des instituteurs de la Suisse romande (dorénavant SIR) à laquelle se rallient les sociétés pédagogiques cantonales. On ne saurait trop relever l'importance considérable de ce regroupement des sociétés cantonales en un faisceau unique, quand on sait combien la prééminence des cantons en matière d'instruction est prégnante sur le territoire helvétique.

Il faut également souligner qu'à peine six mois après cette union régionale, les projets intercantonaux de la SIR se doublent d'une ambition "internationaliste" portée par le pédagogue Alexandre Daguet (1816-1894). Si celui-ci se fait d'abord connaître par son œuvre d'historien et promeut une vision particulariste de la nation suisse, ses conceptions pédagogiques s'avèrent très clairement universalistes ${ }^{9}$. Né à Fribourg - un des bastions du catholicisme helvétique -, Daguet se construit une pensée teintée de libéralisme et de patriotisme en rupture avec les enseignements des jésuites qui assurent sa première formation. Jeune homme, il fréquente le pédagogue Grégoire Girard (1765-1850) qui l'initie à l'universalisme kantien et aux idées de tolérance religieuse. Il baigne dans le cosmopolitisme de la cellule du moine pédagogue. Devenu son assistant vers 1837, au moment où il entre dans le professorat, il accueille les grands noms de la pédagogie européenne qui viennent étudier la déclinaison du mutualisme développée par Girard $^{10}$. Convaincu du caractère

8 Ernest Savary, La Société pédagogique de la Suisse romande (1864-1914). Notice historique à l'occasion du jubilé cinquantenaire de cette société, Lausanne, Imprimeries réunies, 1914, p.7.

9 Sur cette dialectique entre nationalismes et internationalismes, je renvoie à Anne-Marie Thiesse, "Nations, internationalismes et mondialisation", art. cit., p. 15-27.

10 Sur l'itinéraire de ce pédagogue suisse, voir Alexandre Fontaine, Alexandre Daguet (1816-1894) : 
universel de la pédagogie, Daguet et quelques membres fondateurs de la SIR profitent de l'Exposition universelle de 1867 pour intégrer la Suisse romande dans le concert pédagogique européen.

\section{2 - Un projet universaliste aux racines franco-romandes}

En mars 1865, le président de la SIR Frédéric Villommet interpelle son collègue parisien M. Pilate, membre de la conférence des institutrices et instituteurs protestants de la Seine : "Pourquoi ne pourrions-nous pas avoir des congrès internationaux aussi bien que les économistes, les légistes, les ouvriers. Nous aimerions à vous voir prendre l'initiative [...] Il va sans dire que la première réunion aurait lieu à Paris " ${ }^{11}$. Par ces mots, Villommet donne l'impulsion à un projet d'association internationale qui répond à plusieurs préoccupations. La guerre austro-prussienne et les tensions entre la France et la Prusse suite à la victoire de celle-ci à la bataille de Sadowa poussent en effet l'Europe scolaire à s'interroger sur les vertus pacificatrices de l'éducation.

En août 1866 à Fribourg, les instituteurs romands rassemblés lors de leur premier congrès pédagogique discutent de l'introduction de manuels uniques dans les divers cantons qui forment la Suisse française ainsi que du rôle de l'intuition dans l'enseignement élémentaire. Ils officialisent également le projet d'envoyer une délégation à Paris lors de l'Exposition universelle afin d'y étudier l'exposition scolaire et de susciter la création d'une association internationale d'instituteurs. Les principaux acteurs de l'école primaire romande composent la délégation présidée par Daguet. Tous collaborent à L'Éducateur, l'organe des instituteurs romands qui paraît dès janvier 1865 et dont Daguet est le fondateur et directeur. La plupart d'entre eux jouissent déjà de leur propre réseau avec le monde pédagogique français. Jules Paroz (1824-1906), ancien élève de Daguet et créateur de l'école normale évangélique de Grandchamp, a vu sa revue L'Éducateur populaire, fondée en 1848, absorbée par L'École normale de Pierre Larousse dès $1859^{12}$. Il profite d'ailleurs de son séjour parisien pour lancer la publication de son Histoire de la pédagogie chez Charles Delagrave. Daguet a

racines et formation d'un historien libéral-national oublié, mémoire de licence, université de Fribourg, 2005. En ligne : <http://unige.academia.edu/AlexandreFontaine>

11 Lettre du 15 mars 1865, tirée de Rapports sur l'exposition scolaire de Paris en 1867, Lausanne, Imprimerie Borgeaud, 1868, p. V.

12 Voir Jules Paroz, Mémoires d'un octogénaire, Porrentruy, Éditions du Pré-Carré, 1981, p. 161 ainsi que le compte-rendu de Pierre Caspard dans Histoire de l'éducation, nº 13, décembre 1981, p.95-96. 
déjà rencontré Pierre-Philibert Pompée ${ }^{13}$ - le principal interlocuteur des Suisses à Paris - lorsque celui-ci visita les écoles de Girard à Fribourg. Il entretient également une correspondance suivie avec des personnalités françaises de premier plan : le pacifiste Frédéric Passy, le promoteur de l'abolition de la peine de mort Charles Lucas, Jean Macé, le fondateur de la Ligue de l'enseignement et rédacteur du Magasin d'éducation et de récréation (1864) qu'il rencontrera d'ailleurs à Neuchâtel en 1870, ou Gustave Vapereau qui lui consacre une notice bibliographique dans L'année littéraire de $1868^{14}$. Il fréquente Édouard Charton qui le visitera à Neuchâtel en 1873 et publie plusieurs articles consacrés à Girard ou de la plume de Daguet dans le Magasin pittoresque ${ }^{15}$. Plus encore, ce dernier est engagé comme professeur d'histoire nationale par l'Académie de Neuchâtel en même temps que Ferdinand Buisson qui lui offre la direction des articles consacrés à la Suisse du fameux Dictionnaire de pédagogie et d'instruction primaire ${ }^{16}$ et lui propose même de le rejoindre à Paris pour créer une "œuvre internationale d'éducation "17. Si Daguet repousse l'invitation, c'est un autre Helvète, le socialiste libertaire James Guillaume, qui s'exile à Paris en mai 1878 pour devenir la cheville ouvrière du Dictionnaire. Ainsi, est-il besoin de le souligner davantage, la pédagogie européenne se construit au travers d'une multitude d'entreprises collectives et de décisions individuelles, rendues possibles grâce à la mise en place de réseaux qui ont favorisé l'importation massive de méthodes et d'objets pédagogiques élaborés internationalement ${ }^{18}$.

13 Pierre-Philibert Pompée (1809-1872) fut le directeur de l'école (primaire supérieure) municipale Turgot à Paris et le fondateur de l'école professionnelle d'Ivry. Médiateur dans l'espace franco-suisse, il étudia en Suisse la méthode Pestalozzi et rencontra le Père Girard (et Alexandre Daguet) à Fribourg. Lors de l'Exposition universelle de 1867, il fut désigné, grâce à ses bons rapports avec Victor Duruy, pour faire partie du jury international des récompenses comme membre de la classe 90.

14 Je renvoie à l'importante correspondance entretenue par Daguet avec ces personnalités françaises, accessible aux Archives de l'État de Neuchâtel, Fonds Daguet.

15 Édouard Charton, "Biographie de Grégoire Girard ", Le Magasin pittoresque, 1850, p. 220-222 et 1851 , p. 210-212; Alexandre Daguet, "Barbe Schinner, héroïne de la charité à Fribourg (1746-1816)", Le Magasin pittoresque, 1875, p. 186-187; Édouard Charton, "Consultation d'un maître d'école", Le Magasin pittoresque, 1883, p. 189-190.

16 Voir Patrick Dubois, Le Dictionnaire de Ferdinand Buisson. Aux fondations de l'école républicaine (1878-1911), Berne, Peter Lang, 2002; Daniel Denis, Pierre Kahn (dir.), L'école républicaine et la question des savoirs. Enquête au cœur du Dictionnaire de pédagogie de Ferdinand Buisson, Paris, CNRS Éditions, 2003.

17 Alexandre Daguet, "Rapport sur la marche de l'Éducateur", L'Éducateur, nº 17, 1882, p. 259.

18 Sur les enjeux liés à ces réseaux transnationaux, voir notamment Damiano Matasci, L'école républicaine et l'étranger. Une histoire internationale des réformes scolaires en France, 1870-1914, Lyon, ENS Éditions, 2015; Joëlle Droux, Rita Hofstetter (dir.), "Internationalisation in Education: Issues, Challenges, Outcomes", Paedagogica Historica, vol.50, n 1-2, 2014; Gita Steiner Khamsi, Florian 
Le 22 août 1865 à Paris, la délégation romande assiste, sur l'invitation du ministre Duruy, à l'une des fameuses conférences de la Sorbonne organisées pour le perfectionnement des instituteurs français présents à Paris lors de l'exposition universelle. Daguet fait ressurgir les liens franco-suisses, avant d'argumenter les fondements universels et les enjeux de son projet:

"Il y a entre nous, Messieurs et chers amis, plus d'un lien, plus d'un motif de rapprochement. Sans parler de la communauté de langue et du légitime pouvoir qu'exerce sur les esprits la riche et belle littérature française, qui est aussi la nôtre, n'y a-t-il pas pour tous les instituteurs identité de but, d'efforts, d'aspirations? Ne désirons-nous pas tous former une jeunesse intelligente, éclairée, généreuse, pénétrée du même amour du bien et du beau, du saint enthousiasme de Dieu, de l'humanité et de la patrie? En dépit de toutes les différences que peuvent mettre entre les peuples la nationalité et la diversité des méthodes, un fond commun subsiste; c'est qu'il n'y a qu'une éducation comme il n'y a qu'une humanité»"19.

Daguet conclut par un appel à la formation d'une association internationale, guidée par une Suisse romande alors pensée comme un trait d'union entre les peuples du Nord et du Midi :

"Les grands principes qui unissent les peuples et les hommes n'ont jamais été affirmés avec plus de puissance et plus d'éclat que dans les grandes assemblées françaises qui ont proclamé la liberté et l'égalité civile et politique à la fin du dernier siècle. Ne serait-il pas beau, ne serait-il pas glorieux pour vous et pour nous, Messieurs, et chers collègues, de voir sortir de nos délibérations communes une institution qui fût le trait d'union des divers peuples, et réunit les instituteurs de tous les pays en congrès international. C'est à la fondation de ce congrès que j'ose vous convier et la délégation suisse serait heureuse d'y contribuer pour sa faible part ${ }^{20}$.

Pierre-Philibert Pompée souscrit au projet et, dans le sillage de la proposition suisse, indique par ailleurs vouloir mettre en place une exposition pédagogique internationale permanente à Paris, afin que les instituteurs de France puissent

Waldow, Policy Borrowing and Lending in Education, Londres/New York, Routledge, 2012; Klaus Dittrich, Expert going transnational: education at world exhibitions during the second half of the nineteenth century, PhD, University of Portsmouth, 2010; Eckhardt Fuchs (dir.), Bildung International. Historische Perspektiven und aktuelle Entwicklungen, Würzburg, Ergon Verlag, 2006; David Phillips, Kimberly Ochs (dir.), Educational Policy Borrowings: historical perspectives, Oxford, Symposium Books, 2004.

19 Rapports sur l'exposition scolaire de Paris en 1867, op. cit., p. VIII.

Ibid., p. VIII-IX. 
venir étudier à loisir les progrès susceptibles d'être importés dans leurs classes ${ }^{21}$. Cela étant dit, il faut souligner avec insistance combien les structures nationales sur lesquelles doit reposer cette association internationale sont disparates et inégalement développées, ce qui fait dire à Daguet :

"Peut-être l'idée souriait-elle au fond médiocrement à l'administration française; peut-être aussi ne pouvait-elle rien avoir de pratique dans une assemblée composée d'instituteurs venus de provinces éloignées et qui n'avaient jamais entendu parler d'une institution de ce genre; peut-être encore, et c'est des trois versions la plus probable, les chefs de l'instruction publique en France pensèrent-ils sagement qu'avant de songer à une réunion internationale, c'està-dire des divers peuples, il était nécessaire de commencer par organiser une société générale des instituteurs français ${ }^{22}$.

Soucieuse de présider à la destinée de cette association, la délégation suisse propose de se servir des congrès pédagogiques romands comme point de rencontre des divers délégués qui proviennent de toute l'Europe et même des colonies ${ }^{23}$.

\section{3 - Une entreprise supervisée par la Suisse française}

Remise ainsi à l'ordre du jour du deuxième congrès de la SIR en août 1868 à Lausanne, la question est timidement traitée, d'autant que le spectre du choléra a retenu chez eux la plupart des invités étrangers. Néanmoins, les messages d'appui reçus pour l'occasion laissent à penser que l'entreprise possède déjà un début de réseau, en partie constitué à Paris grâce à l'appui de personnalités comme Jules Simon, Albert Le Roy, André Rousselle, tous trois membres de la Société pour l'instruction élémentaire, Pierre-Philibert Pompée et Adrien Guerrier de Haupt, le rédacteur de L'Union des instituteurs. Notons encore que la Société centrale des instituteurs belges, représentée par Emmanuel Van Driessche ${ }^{24}$,

21 Rapports sur l'exposition scolaire de Paris en 1867, op. cit., p. 137-138.

22 Ibid., p. XIV.

23 Les principaux délégués qui fréquentent les congrès romands et participent à l'élaboration des statuts de l'Association pédagogique universelle sont pour l'Italie, Vincenzo de Castro, professeur émérite de l'université de Padoue et membre du conseil-directeur de l'Association nationale pour l'éducation du peuple ainsi que J.-O. Mellé, professeur de belles-lettres à Turin; pour la Belgique, Jean-Joseph Campion, président de la Fédération des instituteurs belges; pour la France, Charles Defodon, secrétaire de la Société pour l'instruction élémentaire à Paris, MM. Merle et Masson, instituteurs ainsi que François-Joseph Gastu, maire d'Alger et son adjoint d'El Biar; pour la Hollande, J.-F. Humalda, inspecteur de la province de Frise; pour la Grèce, M. Frédéricos, secrétaire du ministère de l'Instruction publique et ses adjoints MM. Lascaris et Grégorias; pour la Russie, M. Smolian, inspecteur des écoles sibériennes et M. Swekowski, professeur à Kiev.

24 Emmanuel Van Driessche (1824-1897), instituteur de formation, devient ensuite enseignant de 
Pierre Wynen, président de la Société des instituteurs privés d'Anvers et JeanJoseph Campion, gérant de la revue pédagogique bruxelloise Le Progrès ainsi que la Societa pedagogica italiana et son président Giuseppe Sacchi s'agrègent à ce premier réseau et se retrouvent lors des assemblées romandes.

Deux ans plus tard, lors du congrès neuchâtelois de 1870, la déclaration de guerre à la Prusse sonne momentanément le glas de toute idée d'union internationale. C'est donc lors des congrès de Genève (1872) et de Saint-Imier (1874) que la question d'une association internationale revient au centre des préoccupations. La vocation internationale dont jouit la "Rome protestante" a une incidence certaine dans la reprise du dialogue et du projet de fondation de l'Association pédagogique universelle. Pourtant, cet optimisme de façade n'efface en rien les obstacles qui surgissent lors de l'assemblée. Les effets de la guerre franco-prussienne se font sentir. Certains, comme l'instituteur vosgien Masson ou le professeur italien Pietro Preda - collègue de Daguet et de Buisson à l'Académie de Neuchâtel -, proposent d'exclure l'Allemagne. Preda soumet l'idée de fonder "pour le moment et en attendant mieux, une Association des instituteurs de race latine, qui comprendrait les pays où l'on parle français, italien et espagnol $»^{25}$. La réponse de Daguet est sans équivoque :

"Ce que nous voulons, ce n'est donc pas une fédération partielle, exclusive. Surtout pas d'exclusion de l'Allemagne qui est avec la Suisse la terre classique de la pédagogie rationnelle et le berceau de l'invention de Gutenberg, sans lequel la diffusion actuelle des lumières eût été impossible. C'est le propre de tous les Nabuchodonosors français et allemands d'être éblouis de leurs victoires. Mais la haine de la France et de l'Allemagne, toute vivace qu'elle soit, ne sera pas éternelle, et si des représentants de ces deux grandes nations doivent se rencontrer et se tendre une main fraternelle, c'est à coup sûr sur le terrain neutre de la science et de l'éducation. [...] Quoi qu'il advienne, ouvrons toutes grandes les portes de l'Alliance pédagogique. Il y aura déjà assez de difficultés de détail, d'obstacles financiers à vaincre. Ceux qui viendront à nous, Français ou Allemands, seront des hommes de cœur, élevés au-dessus des haines de race et de nation. Acceptons-les tous : paix aux hommes de bonne volonté sur la terre ${ }^{26}$.

On mesure ici le caractère pacificateur que Daguet attribue à la pédagogie, dont une des principales fonctions est d'apaiser les tensions internationales.

néerlandais à l'Athénée de Bruxelles. Franc-maçon, il est proche des progressistes et se trouve aussi impliqué dans les activités de la Ligue de l'enseignement.

25 Compte-rendu du IV congrès scolaire de la Société des instituteurs de la Suisse romande tenu à Genève les 29, 30 et 31 juillet 1872, Genève, Impr. Taponnier et Studer, 1872, p. 83.

Ibid., p. 84 . 
Relevons au passage que ce sera également via le canal de la pédagogie que l'inspecteur strasbourgeois Guillaume Jost, invité au XXVI congrès des instituteurs allemands de Darmstadt (décembre 1885), tendra une main réconciliatrice à l'Allemagne : "Nous pouvons différer en politique, mais en ce qui concerne l'éducation populaire, nous ne connaissons pas de frontières. Il n'y a pas de pédagogie spécifiquement allemande, italienne, française et celui-là n'est pas un pédagogue qui croit n'avoir rien à apprendre chez les autres peuples ${ }^{27}$.

Enfin, les discussions sur le nom à donner à l'association s'avèrent houleuses. Plusieurs intitulés sont discutés : "Association universelle des instituteurs", "Société pédagogique universelle", ou encore "Ligue universelle d'enseignement". Daguet défend même l'utilisation délicate de "Société internationale" et s'attache à faire taire certaines rumeurs suggérant "qu'en Italie, on a cru qu'il s'agissait d'une espèce d'internationale poursuivant des idées révolutionnaires ${ }^{28}$. L'assemblée aboutit finalement à un consensus et ses membres proclament, à l'unanimité, la création de l'Association pédagogique universelle :

"Le congrès scolaire, réuni à Genève le 31 juillet 1872, décide à l'unanimité la création d'une Association pédagogique universelle. Pour atteindre son but, le congrès charge son bureau d'adresser immédiatement un appel aux Sociétés pédagogiques des différents pays. Cet appel sera signé par le bureau du congrès actuel et par les délégués étrangers présents. Dès que trois sociétés de pays différents auront donné leur adhésion au projet de la Société pédagogique universelle, elles désigneront des délégués pour s'entendre sur la constitution même de la Société et pour aviser aux meilleurs moyens d'atteindre le but ${ }^{29}$.

Cependant, deux ans plus tard, dans un rapport qu'il rédige pour préparer les débats du congrès de Saint-Imier de 1874, l'instituteur vosgien Masson réitère son vœu de voir naître une association des membres du corps enseignant "de race latine". Son collègue M. Vion appelle quant à lui à la création d'un bulletin élaboré sous l'égide de la France et voué à terme à devenir l'organe de l'enseignement laïque. Selon lui, un grand congrès constituant pourrait avoir lieu l'année suivante à Pâques, à Paris, à l'occasion du congrès de géographie ${ }^{30}$. On se trouve ici en face d'une des rares contestations du leadership romand, que Daguet va étouffer d'une manière plutôt autoritaire, sinon arrogante :

27 Alexandre Daguet, "Pédagogie de l'Allemagne", L'Éducateur, nº 1, 1886, p. 6.

28 Compte -rendu du V e congrès scolaire..., op. cit., p. 98.

29 Compte -rendu du IV eongrès scolaire..., op. cit., p. 88.

30 Compte -rendu du V e congrès scolaire..., op. cit., p. 91. 
"Nous aimerions bien pouvoir partager la confiance de M. Vion quant à la part que prendra son pays à notre œuvre, mais il nous semble que la question de débuter par la France est un peu prématurée et même hasardée. Il sera donc plus prudent, à notre avis, de tenir ailleurs le premier congrès dont il s'agit. Il ne suffit pas que l'esprit d'association existe dans un pays; il faut de plus qu'il soit encouragé et non comprimé. Nos collègues de la France, bien disposés d'ailleurs à nous prêter un concours efficace, ne le pourront que par un zèle soutenu et une grande persévérance à réclamer pour eux le droit de réunion qu'on leur conteste, et qui seul peut donner essor à leurs légitimes aspirations $»^{31}$.

Le congrès de Saint-Imier de 1874 marque ainsi le début des désillusions pour Daguet, qui ne dissimule plus son irritation. Le troisième jour, au moment d'aborder la question internationale, le temple où se tient le congrès se vide de ses participants : "Soit que cette question n'intéressa pas au même degré que les autres, soit qu'on trouva trop long le terme de trois jours, le temple était presque désert quand on traita ce sujet et l'auditoire réduit à 40 ou 50 personnes", peut-on lire dans le Journal de Genève du 29 juillet 1874. Lorsqu'on s'essaie timidement à une ébauche de statuts, la montée des intérêts nationaux, les intérêts particuliers marquent un coup d'arrêt définitif au projet universaliste romand. Les tractations s'enlisent, n'aboutissent finalement qu'à des résultats très mitigés. Enfin, durant les préparatifs pour le congrès de Fribourg de 1877, l'inactivité de la commission chargée de traiter la question pousse Daguet à prendre acte de cet échec. Cette entreprise s'avère en fait, selon lui, "un idéal impossible à réaliser dans les circonstances présentes, ou comme une utopie généreuse éclose au souffle exhilarant et enthousiaste des expositions universelles ${ }^{32}$.

En dépit de cet échec, cette association va perdurer sous une autre forme : à cette tentative avortée de rassemblement "physique" se substitue une communauté fort puissante à travers les publications. Le réseau occidental de la pédagogie libérale-nationale va dès lors, selon la fameuse formule de Norbert Elias, "étendre ses chaînes d'interdépendance " ${ }^{33}$ à l'emploi massif de la presse et des revues afin de traduire, transférer ${ }^{34}$ et diffuser les éléments d'un système éducatif pensé de manière collective et de ce fait de plus en plus standardisé.

31 Ibid.

32 Alexandre Daguet, "Un mot sur la question de la Fédération universelle», L'Éducateur, nº 9, 1876, p. 135-136.

33 Norbert Elias, La société des individus, Paris, Fayard, 1991.

34 Sur l'usage de la méthodologie des transferts culturels en histoire de l'éducation, voir Alexandre Fontaine, Aux heures suisses de l'école républicaine. Un siècle de transferts culturels et de déclinaisons pédagogiques dans l'espace franco-romand (1800-1900), Paris, Demopolis, coll. Quaero, 


\section{II - «L'association est morte, vive l'association!»}

C'est dans cette perspective que Daguet s'attelle à la constitution d'un réseau européen de la presse pédagogique, qu'il appelle ainsi de ses voux :

"Si la fédération universelle par voie de congrès et de délégués est difficile à établir et à pratiquer, il n'en est pas de même de la Fédération qui s'opère par la voie des journaux et de l'échange d'idées qu'elle établit entre les éducateurs et écrivains pédagogiques non seulement de l'Europe, mais des deux hémisphères. C'est à cet échange surtout que nous pensions dès l'origine de notre Société et son organe, et nous n'avons rien négligé pour le créer, l'étendre à tous les peuples civilisés, à commencer par ceux dont la langue est généralement comprise et même à ceux dont la langue n'est connue que de quelques initiés. Les idées lumineuses ne sont pas l'apanage d'une seule nation ou de certains peuples, pas plus que d'un ou de plusieurs individus. Il ne faut rien mépriser, rien négliger. Puis, vous pouvez être utiles et apporter des lumières à ceux qui ne vous en donneraient pas. Quoi de plus beau d'ailleurs, de plus profitable et de plus touchant que ces relations de peuple à peuple, d'esprits à esprits, de corps enseignant à corps enseignant, d'un bout du monde à l'autre? Si l'humanité doit jamais former une grande famille, c'est assurément par l'école que doit commencer cette fusion des divers groupes de l'humanité qu'on nomme les nations ${ }^{35}$.

Ainsi, dès le milieu des années 1870, Daguet propose régulièrement dans L'Éducateur une "Revue de la pédagogie européenne», c'est-à-dire des pays avec lesquels son journal est en relation par l'échange de leurs numéros. Si la pratique du "courrier international" n'est pas neuve et fut déjà pratiquée antérieurement par d'autres revues, les acteurs de ce réseau font de la presse pédagogique un point de rencontre et un élément médiateur fondamental de la diffusion d'une pédagogie libérale-nationale - essentiellement anticléricale - dont on peut reconstituer la constellation à partir du réceptacle incarné par L'Éducateur.

2015; Christine Mayer, "Female education and the cultural transfer of pedagogical knowledge in the eighteenth century ", Paedagogica Historica, vol. 48, nº 4, 2012, p. 511-526; Danièle Tosato-Rigo, "Une didactique des droits de l'homme? Autour de quelques catéchismes républicains helvétiques ", in Droits de l'homme et constitution moderne. La Suisse au tournant des XVIII ${ }^{e}$ et XIX ${ }^{e}$ siècles, Genève, Slatkine, 2012, p.276-295; Jean-François Goubet, Des maîtres philosophes? La fondation de la pédagogie générale par l'Université allemande, Paris, Garnier, 2012; Gita Steiner-Khamsi, Florian Waldow, Policy Borrowing and Lending in Education, World Yearbook of Education, Londres/New York, Routledge, 2012; Monique Mombert, L'enseignement de l'allemand en France 1880-1918. Entre "modèle allemand" et "langue de l'ennemi", Strasbourg, Presses Universitaires de Strasbourg, 2001.

35 Alexandre Daguet, "Revue de la presse pédagogique en Europe et aux États-Unis et relations de L'Éducateur à l'étranger", L'Éducateur, nº 19, 1876, p. 290. 


\section{1 - Les étapes d'un réseau européen}

\section{de la presse pédagogique libérale-nationale}

Dès ses premières semaines de publication en 1865, L'Éducateur était entré en relation avec la revue Patria e Famiglia du pédagogue lombard Giuseppe Sacchi, avec El Magisterio Espanol dirigé par le pédagogue Emilio Ruiz de Salazar ainsi qu'avec Los Annales de primera ensenaza rédigées par Mariano Carderera, directeur de l'instruction publique au ministère du Fomento à Madrid et par ailleurs rédacteur dans le premier Dictionnaire de pédagogie de Buisson ${ }^{36}$.

Grâce à la traduction, plusieurs articles qui le plus souvent sont choisis pour légitimer des politiques scolaires locales jouissent d'une diffusion européenne et facilitent la circulation de savoirs pédagogiques dans l'espace occidental ${ }^{37}$. Ces processus de transferts laissent apparaître des situations d'osmose et d'assimilation de savoirs qui transitent via les revues pour être adaptés par leur contexte d'accueil ${ }^{38}$. Comme le relève Daguet, "les feuilles étrangères ne se bornent pas à échanger avec nous; elles nous citent souvent, empruntent ou traduisent nos articles ${ }^{39}$. L'Allgemeine Deutsche Zeitung de Darmstadt réserve une place de choix aux articles de fond de L'Éducateur. Le très anticlérical Christian Jessen reproduit in extenso plusieurs articles romands dans ses Freien Pädagogischen Blätter publiées à Vienne. Dans les années 1871-1872, L’Éducateur entre en relation avec deux revues anglaises, le School Board Chronicle et l'organe de la National Education League, toutes deux imprimées à Londres. Dès lors, comme le souligne Daguet en 1872 :

"Il ne manque plus au faisceau de nos relations extérieures que les États-Unis avec lesquels L'Éducateur n'a fait jusqu'ici de fugitifs échanges, et avec lesquels, cependant, il est à désirer qu'il s'établisse des rapports plus suivis, ne fût-ce que pour naturaliser en Europe les livres élémentaires de l'Union américaine,

36 Voir Patrick Dubois, Le Dictionnaire de pédagogie et d'instruction primaire de Ferdinand Buisson. Répertoire biographique des auteurs, Paris, INRP, 2002, p. 51.

37 Voir Béatrice Haenggeli-Jenni, Alexandre Fontaine, Patrick Bühler, "Une circulation des idées pédagogiques sur papier. Presse d'éducation, transferts et trajectoires transnationales des savoirs (1850-2000) ", numéro thématique de la Revue suisse des sciences de l'éducation, vol. 36, $\mathrm{n}^{\circ}$ 1, 2014.

38 Voir Michel Espagne, "La notion de transfert culturel", Revue Sciences/Lettres, n 1, 2013 et, du même, L'Ambre et le fossile. Transferts germano-russes dans les sciences humaines XIX ${ }^{e}$-XX ${ }^{e}$ siècles, Paris, Armand Colin, 2014.

39 Alexandre Daguet, "Les jardins de l'enfance et l'école de Chantepoulet à Genève. Conférences pédagogiques données par M. Sante Polli, directeur de l'École normale de Milan", L'Éducateur, n 14 , 1868, p. 220- 223. 
dont quelques-uns nous ont paru bien conçus et unissaient, à un degré peu commun, le côté pratique au côté idéal $\rrbracket^{40}$.

L'année suivante, L'Éducateur s'associe d'ailleurs au Journal of Education de Boston. Ces échanges transnationaux, progressifs, suscitent la mise en place d'un réseau spécifique des revues pédagogiques, un lobby international regroupant les représentants d'une pédagogie éclectique, spiritualiste et anticléricale (tableau ci-dessous).

\begin{tabular}{l|l|l}
\hline Revues & Rédacteurs & $\begin{array}{l}\text { Orientations } \\
\text { des rédacteurs }\end{array}$ \\
\hline L'Éducateur & A. Daguet & Catholique libéral, maçon \\
\hline Schweizerische Lehrerzeitung & J.-U. Rebsamen & Protestant libéral \\
\hline L’Educatore della Svizzera italiana & G. Ghiringhelli & Chanoine radical \\
\hline Le Progrès de Bruxelles & J.-J. Campion & Libre penseur, maçon \\
\hline $\begin{array}{l}\text { Manuel général de l'instruction } \\
\text { primaire }\end{array}$ & C. Defodon & Catholique libéral \\
\hline Revue pédagogique & F. Buisson & Protestant libéral \\
\hline Patria e Famiglia & G. Sacchi & Patriote italien, libéral \\
\hline $\begin{array}{l}\text { Enrico Pestalozzi o l'Educazione } \\
\text { nuova }\end{array}$ & V. di Castro & Patriote italien, libéral \\
\hline Freien pädagogischen Blätter & C. Jessen & Libéral anticlérical \\
\hline School Board Chronicle (London) & A. Bikkers & Libéral, maçon \\
\hline De Vekker (La Haye) & M. Lallemand & Libéral \\
\hline Los Annales de primera ensenaza & M. Carderera & Libéral \\
\hline El Magisterio Espanol & E. Salazar & Libéral \\
\hline
\end{tabular}

Revues européennes en relation avec L'Éducateur (1865-1890)

\section{2 - Les liens particuliers avec la France}

La communauté de langue entre la Suisse romande et la France va de fait privilégier les échanges et les métissages pédagogiques entre les deux espaces ${ }^{41}$. Peu après son retour à Paris en 1870, Buisson fait la publicité de L'Éducateur dans le Bulletin pour l'enseignement élémentaire de Paris :

"L’Éducateur est une solide et consciencieuse revue pédagogique publiée par les instituteurs de la Suisse romande. Parmi les nombreuses feuilles que nous

40 Compte-rendu du IV e congrès scolaire..., op. cit., p. 52-53.

41 Sur la notion de métissage, voir Serge Gruzinski, La pensée métisse, Paris, Fayard, 2012 et Laurier Turgeon (dir.), Regards croisés sur le métissage, Laval, Les Presses de l'Université, 2003. 
parcourons, aucune ne contient des articles plus substantiels. Comme le Progrès, l'Éducateur n'est pas exclusif; il recueille de tous les côtés les renseignements, les faits, les livres, les méthodes : aussi est-il d'une lecture tout à fait profitable pour l'instituteur qui aime sa profession et qui s'intéresse à tout ce qui se fait, au près et au loin, pour l'éducation du peuple ${ }^{42}$.

De son côté, Daguet fait savoir dans L'Éducateur que les ministres de l'Instruction publique et des Cultes qui se sont succédé en France de Victor Duruy à Jules Simon, ont gratifié sa revue de l'envoi régulier du Bulletin administratif de leur département ministériel ${ }^{43}$. Comme souvent en Suisse, on cherche à légitimer les entreprises locales par l'approbation symbolique et le cachet de l'étranger. C'est dans cette perspective que le comité de rédaction de L'Éducateur adresse sa revue à plusieurs personnalités françaises. Victor Hugo accuse réception d'Hauteville-House le 18 avril 1869 :

"Absorbé par des travaux urgents, je n'ai pu vous remercier plus tôt de votre excellente lettre. Voir le peu que je fais si bien compris, et par de telles intelligences, serait pour moi une douce récompense, si une récompense m'était due; mais je suis loin de le penser. Le devoir, c'est là ce que je tâche de faire. Hors de là, je ne suis rien. Vous m'envoyez votre utile journal, je le lis avec intérêt et je vous prie de croire à toute ma cordialité $"^{44}$.

En outre, de nombreux articles de L'Éducateur sont reproduits dans des revues pédagogiques françaises : dans le Manuel général de l'instruction publique de Charles Defodon, dans l'Union des instituteurs d'Adrien Guerrier de Haupt ${ }^{45}$ ou dans le Bulletin pour l'enseignement élémentaire de Paris. Mais à l'évidence, c'est Ferdinand Buisson qui va s'attacher à faire connaître L'Éducateur en France et même beaucoup plus loin :

"Je vous avais demandé l'article Éducateur pour le 29 avril. Si vous pouviez m'envoyer d'ici à 8 jours à défaut de cet article une note d'une page ou deux au plus sur l'origine du journal, ses fondateurs, son mode de publication, ses principales séries de travaux pédagogiques, etc., je pense qu'il vous serait agréable de profiter d'une bonne occasion que je puis vous offrir. Je ferais imprimer cet article-là dans un nouveau spécimen que je vais faire adresser à tous les journaux pédagogiques dont je sais l'existence en Europe et en

42 Ferdinand Buisson, "L'Éducateur de la Suisse romande", Bulletin pour l'enseignement élémentaire de Paris, juin-juillet 1871.

43 Alexandre Daguet, "Revue de la presse pédagogique en Europe et aux États-Unis, et relations de l'Éducateur avec l'Étranger", L'Éducateur, nº 19, 1876, p. 294-295.

44 Réponse de Victor Hugo à Monsieur Auguste Biolley, président de la SIR, in Compte-rendu du Congrès pédagogique de Neuchâtel en 1870, et Rapport sur l'exposition scolaire de la Suisse romande, Neuchâtel, Impr. G. Guillaume Fils, 1870, p.37-38.

45 Le congrès scolaire de Lausanne. Rapport sur la troisième session de l'assemblée générale des Instituteurs de la Suisse romande réunis les 5 et 6 août 1868, Lausanne, Impr. Borgeaud, p. 8. 
Amérique. Ce serait donc un moyen de notoriété assez bon mis à votre portée. Vous me comprenez bien $" 46$.

Buisson s'intéresse particulièrement aux problématiques romandes développées dans les articles de fond de la revue et dans ce sens concède à Daguet : "Je n'ai pas besoin de vous dire que de mon côté je serais heureux d'avoir l'occasion, dans mon service d'inspecteur, de faire connaître et apprécier ici une publication où les Français en particulier auraient tout à apprendre $»^{47}$. Ce sont d'ailleurs ces mêmes réseaux que Ferdinand Buisson va réactiver et utiliser afin de lancer son propre projet international.

\section{3 - La réactivation de Ferdinand Buisson}

Il est intéressant de constater que Buisson, au fait d'une véritable pensée sur l'éducation internationale ${ }^{48}$, entre en scène au moment où son ancien collègue suisse s'apprête à mettre un terme définitif à son projet universaliste. En 1877, il écrit en effet à Daguet :

"Une lettre de M. Campion m'encourage à faire auprès de vous une démarche qui ne sera peut-être pas plus heureuse que les précédentes. Il s'agit de ce fameux projet de société ou alliance internationale des instituteurs et amis de l'enseignement. Ce projet est né je crois à Genève, est-il enterré? Si oui n'en parlons plus. Sinon, y aura-t-il à Fribourg cette année une séance consacrée à l'étude de la question? Vous savez que j'avais songé à la création d'une société (internationale) d'études pédagogiques.

[...] Il parait que les instituteurs de la Suisse romande et vous leur grand inspirateur vous ne croyez plus devoir encourager ce projet. Soit. Peut-être la chose ne se fera-t-elle pas! Du moment qu'une voix aussi importante que celle de la Suisse manque à ce concert, il n'y a qu'à renoncer à l'idée. D'ailleurs nous autres Français nous ne demandons ni ne songeons, quoique nous ayons peut-être une autre réputation en Suisse, à nous annexer les sociétés étrangères. Au contraire si vous créez votre société ou alliance universelle, nous serons bien aisés d'y rentrer à la place qu'il vous plaira de nous assigner. Nous travaillons en ce moment à une certaine suite et dans un esprit pratique à l'amélioration de nos institutions scolaires. Et nous éprouvons le besoin de nous instruire, de nous informer, de nous tenir au courant de ce qui se fait de bon au près et au loin.

46 Lettre de Ferdinand Buisson à Alexandre Daguet, sans lieu ni date, Archives de l'État de Neuchâtel, Fonds Daguet.

47 Ibid.

48 Ferdinand Buisson, L'instruction et l'éducation internationale, Paris/Berne, Bureau de la Grande Revue/Ligue internationale de la paix et de la liberté, 1905. 
Si donc il était possible de profiter du congrès de Fribourg pour un rapprochement à titre quelconque, fut-il même borné à l'établissement d'un échange régulier de communications et de renseignements entre les écoles de divers pays, je me ferais un plaisir d'aller à ce congrès et d'en suivre les travaux, si toutefois les étrangers peuvent y assister?»"

Buisson avait déjà réfléchi à la constitution d'une organisation internationale. Suite au premier congrès de la Ligue de la paix et de la liberté convoqué à Genève en septembre 1867, il souhaitait faire de cette instance "une sorte de société internationale pour l'organisation universelle de l'éducation démocratique ${ }^{50}$. Si la réponse de Daguet nous est inconnue, il semble que celui-ci ait interprété les mots de Buisson comme une ingérence et réagi contre cette volonté, de la part de son ancien collègue, de renverser la situation à son avantage comme Buisson l'évoque dans une autre lettre :

"Vous vous méprenez sur le sens de ma proposition, si vous y voyez rien qui puisse donner ombrage à L'Éducateur et à la Société suisse. Au contraire, il s'agit de répondre à l'appel international de Genève et de Saint-Imier et de fonder quelque chose en conséquence d'un commun accord. Je vous en écrirais plus long sur ce sujet que je crois de nature à vous intéresser si je ne venais de voir ici M. Sandoz, qui est au courant de mes vues et intentions. Il vous en parlera et j'espère vous convaincra qu'il y a une envie bonne et utile à faire, où la Suisse ne peut refuser son concours à la France $\aleph^{51}$.

Ces échanges, marqués en filigrane par de tenaces batailles d'egos, ne se soldent pas par quelque réalisation concrète. Buisson s'essaiera une seconde fois à la réactivation du projet au tournant du XIX ${ }^{e}$ siècle, dans le sillage de la Fédération des amicales de France créée en 1900, qui précède et constitue par ailleurs un terrain favorable à l'émergence du syndicalisme enseignant ${ }^{52}$.

Dans le Manuel général de l'instruction primaire du 13 juin 1903, un comité d'action constitué pour l'occasion dévoile le programme d'une excursion

49 Lettre de Ferdinand Buisson à Alexandre Daguet, sans lieu ni date, mais il s'agit assurément d'une lettre envoyée dans le courant de 1877, année du congrès de Fribourg, Archives de l'État de Neuchâtel, Fonds Daguet.

50 Ferdinand Buisson, "L'abolition de la guerre par l'instruction", Les États-Unis d'Europe, n 17, 26 avril 1868, p. 66 .

51 Lettre de Ferdinand Buisson à Alexandre Daguet, sans lieu ni date (courant 1877), Archives de l'État de Neuchâtel, Fonds Daguet.

52 Voir Loïc Le Bars, "Amicales et syndicats dans l'enseignement primaire (1901-1919)", in Danielle Tartakowsky, Françoise Tétard, Syndicats et associations. Concurrence ou complémentarité? Rennes, Presses universitaires de Rennes, 2004, p.37-46. 
des membres de l'enseignement primaire français en Helvétie ${ }^{53}$. Celle-ci doit marquer le début des grandes excursions pédagogiques en Europe, patronnées notamment par Ferdinand Buisson, Gabriel Compayré et Émile Levasseur. Ce qu'il convient surtout de souligner, c'est que ces organisateurs avaient pour intention de provoquer la constitution d'un "comité international d'études pédagogiques" afin d'établir des liens entre tous les instituteurs d'Europe. Ernest Savary, auteur d'une notice historique rédigée à l'occasion du jubilé cinquantenaire de la Société pédagogique de la Suisse romande, souligne à cet égard que "pendant l'été 1903, les instituteurs de la Suisse romande reçurent la visite d'une caravane de leurs collègues français qui venaient visiter notre pays et nous proposer la création d'une Union internationale d'instituteurs. Le projet fut accueilli avec empressement par les dirigeants de notre association mais, rentrés dans leurs foyers, les maitres français oublièrent complètement ce qu'ils nous avaient proposé! „\$4 . En dépit de cette nouvelle déconvenue, ces diverses expériences de rapprochements internationaux constitueront un socle sur lequel les acteurs de l'éducation occidentale pourront s'appuyer après le premier conflit mondial pour réactiver et parachever cette lente mise en réseau, non sans provoquer d'ailleurs de substantiels avancements au niveau local.

\section{Conclusion : échec global, avancées locales}

L'analyse de ces tentatives manquées permet de mieux comprendre les raisons des échecs constitutifs du mouvement associatif européen du second XIX ${ }^{\mathrm{e}}$ siècle. Tout d'abord, le manque de moyens financiers a lourdement pesé sur l'avenir de l'Association pédagogique universelle. Ensuite, il eut été nécessaire que tout pays qui souhaitait y prendre part disposât déjà d'un réseau de sociétés cantonales, régionales ou départementales. On l'a vu, Buisson ne s'est concrètement engagé dans le mouvement international qu'après la création de la Fédération des amicales de France (1900) et l'avènement de structures locales et régionales sur lesquelles il pouvait légitimement reposer ses ambitions ${ }^{55}$.

53 "Excursion des membres de l'enseignement primaire en Suisse du 5 au 11 septembre 1903 ", Manuel général de l'instruction primaire, $\mathrm{n}^{\circ} 24,13$ juin 1903, p. 282.

54 Ernest Savary, La Société pédagogique de la Suisse romande..., op. cit., p. 35-36.

55 Sur l'histoire des amicales et du syndicalisme enseignant, voir M. T. Laurin [Maurice Tortillet], Les instituteurs et le syndicalisme (amicales et syndicats d'instituteurs), Paris, Librairie des sciences politiques et sociales, 1908; Frédéric Mole, L'école laïque pour une République sociale. Controverses pédagogiques et politiques (1900-1914), Rennes, Presses universitaires de Rennes, 2010; Bruno 
Enfin, il faut pointer la multiplicité des "internationales de l'éducation" qui, pour survivre, se livrent tout au long du second XIX ${ }^{e}$ siècle une concurrence acharnée. Rappelons qu'en 1868, le professeur Hermann Karl von Leonhardi et la frobélienne $\mathrm{M}^{\mathrm{me}}$ de Marenholtz-Bülow fondent l'Allgemeine Erziehungsverein dans le but d'engendrer une Confédération internationale des amis de l'éducation $^{56}$. Cette concurrence s'accroît encore avec la création d'une "Société internationale", composée d'une cinquantaine d'instituteurs suisses, badois et bavarois. Fondée le 18 mai 1872 à Rorschach dans le canton de Saint-Gall, cette association est appuyée par la Schweizerische Lehrerzeitung (Revue des instituteurs de Suisse allemande) et s'incarne de fait comme le contrepoids germanique de l'entreprise romande.

Toutefois, et malgré l'échec de l'Association pédagogique universelle, on prend conscience en France de l'importance des réunions internationales et de la nécessité de rassembler les instituteurs dans des structures départementales, regroupées ensuite dans un faisceau national. L'idée fait son chemin et, en 1885, le maire du Havre Jules Siegfried convoque un congrès international d'instituteurs. Mais c'est davantage au plan national que ce rassemblement permet des avancées, lesquelles seront poursuivies lors du congrès convoqué à Paris en 1887. Une résolution tendant à la constitution, dans chaque département, d'une société autonome et amicale des instituteurs y est votée, la fédération de ces sociétés devant aboutir à l'Union amicale des instituteurs de France ${ }^{57}$.

Il faut souligner que ce mouvement français de fédération départementale, combattue par le ministre Eugène Spuller au nom de la souveraineté et de l'unité nationales, s'inspire des sociétés pédagogiques belges et romandes, auxquelles le syndicaliste Gustave Francolin consacre plusieurs notices dans Les congrès d'instituteurs ${ }^{58}$. Née des discussions du Havre, cette feuille paraît dès

Poucet, "Association et syndicalisme dans l'enseignement privé dans la première moitié du XXe siècle", in Danielle Tartakowsky, Françoise Tétard, Syndicats et associations..., op. cit., p. 107-116 et, dans le même volume, Jean-Paul Martin, "Ligue de l'enseignement, amicales et syndicats d'instituteurs sous la Troisième République", p. 361-370.

56 L'Allgemeine Erziehungsverein se met en place lors des congrès philosophiques de Prague (1868) puis de Francfort l'année suivante. Stoppée pour un temps par la guerre franco-allemande, la constitution de l'association fut reprise et finalisée lors d'un congrès convoqué à Dresde en mai 1871.

57 Voir l'article "Congrès d'instituteurs, congrès pédagogiques", in Ferdinand Buisson (dir.), Nouveau Dictionnaire de pédagogie et d'instruction primaire, en ligne sur <http://www.inrp.fr/editionelectronique/lodel/dictionnaire-ferdinand-buisson >

58 Les congrès d'instituteurs. Comptes-rendus des congrès pédagogiques internationaux, nationaux et régionaux, des conférences cantonales, des associations syndicats, unions et fédérations d'instituteurs (gérant : G. Francolin), Paris, Impr. L. Hugonis, 1885-1889. 
novembre 1885 afin de légitimer la création d'une Fédération des instituteurs français. Il faudra néanmoins attendre l'arrivée au pouvoir des républicains radicaux et le vote de la loi de 1901 sur les associations pour voir les amicales se multiplier en toute légalité59.

Ainsi, la structuration d'une "Internationale de l'éducation" doit aussi se penser au travers de ses maillons successifs antérieurs, même si la plupart d'entre eux se sont soldés par des échecs. Heinz-Gerhard Haupt souligne le danger d'écrire une histoire des interconnexions qui ne prendrait en compte que ses succès $^{60}$. La mise en perspective de cet essai manqué d'Association pédagogique universelle rappelle que la lente constitution des internationalismes éducatifs demeure le fruit d'ardents combats, de concurrences nationales, de rivalités d'egos et de jeux diplomatiques fort subtils.

Alexandre Fontaine

Université de Genève (ERHISE), ENS-Ulm (UMR 8547 "Transferts culturels") alexandre.fontaine@unige.ch

59 Bertrand Geay, Le syndicalisme enseignant, Paris, La Découverte, 2005, p. 32-57 (1 ${ }^{\text {re }}$ éd. 1997).

60 Heinz-Gerhard Haupt, "Une nouvelle sensibilité : la perspective transnationale", Cahiers Jaurès, $\mathrm{n}^{\circ} 200$, avril-juin 2011, p. 178. 